\title{
Markov-switching generalized additive models
}

\author{
Roland Langrock $^{1}$ - Thomas Kneib ${ }^{2}$ - Richard Glennie ${ }^{3}$. Théo Michelot ${ }^{4}$
}

Received: 11 May 2015 / Accepted: 15 December 2015 / Published online: 28 December 2015

(C) The Author(s) 2015. This article is published with open access at Springerlink.com

\begin{abstract}
We consider Markov-switching regression models, i.e. models for time series regression analyses where the functional relationship between covariates and response is subject to regime switching controlled by an unobservable Markov chain. Building on the powerful hidden Markov model machinery and the methods for penalized B-splines routinely used in regression analyses, we develop a framework for nonparametrically estimating the functional form of the effect of the covariates in such a regression model, assuming an additive structure of the predictor. The resulting class of Markov-switching generalized additive models is immensely flexible, and contains as special cases the common parametric Markov-switching regression models and also generalized additive and generalized linear models. The feasibility of the suggested maximum penalized likelihood approach is demonstrated by simulation. We further illustrate the approach using two real data applications, modelling (i) how sales data depend on advertising spending and (ii) how energy price in Spain depends on the Euro/Dollar exchange rate.
\end{abstract}

Keywords P-splines · Hidden Markov model · Penalized likelihood · Time series regression

Roland Langrock

roland.langrock@uni-bielefeld.de

Bielefeld University, Bielefeld, Germany

2 University of Göttingen, Göttingen, Germany

3 University of St Andrews, St Andrews, UK

4 INSA de Rouen, Saint-Étienne-du-Rouvray, France

\section{Introduction}

In regression scenarios where the data have a time series structure, there is often parameter instability with respect to time (Kim et al. 2008). A popular strategy to account for such dynamic patterns is to employ regime switching where parameters vary in time, taking on finitely many values, controlled by an unobservable Markov chain. Such models are referred to as Markov-switching or regime-switching regression models, following the seminal papers by Goldfeld and Quandt (1973) and Hamilton (1989). A basic Markovswitching regression model involves a time series $\left\{Y_{t}\right\}_{t=1, \ldots, T}$ and an associated sequence of covariates $x_{1}, \ldots, x_{T}$ (including the possibility of $x_{t}=y_{t-1}$ ), with the relation between $x_{t}$ and $Y_{t}$ specified as

$Y_{t}=f^{\left(s_{t}\right)}\left(x_{t}\right)+\sigma_{s_{t}} \epsilon_{t}$,

where typically $\epsilon_{t} \stackrel{i i d}{\sim} \mathcal{N}(0,1)$ and $s_{t}$ is the state at time $t$ of an unobservable $N$-state Markov chain. In other words, the functional form of the relation between $x_{t}$ and $Y_{t}$ and the residual variance change over time according to state switches of an underlying Markov chain, i.e. each state corresponds to a regime with different stochastic dynamics. The Markov chain induces serial dependence, typically such that the states are persistent in the sense that regimes are active for longer periods of time, on average, than they would be if an independent mixture model was used to select among regimes. The classic example is an economic time series where the effect of an explanatory variable may differ between times of high and low economic growth (Hamilton 2008).

The simple model given in (1) can be (and has been) modified in various ways, for example allowing for multiple 
covariates or for general error distributions from the generalized linear model (GLM) framework. An example for the latter is the Markov-switching Poisson regression model discussed in Wang and Puterman (2001). However, in the existing literature the relationship between the target variable and the covariates is commonly specified in parametric form and usually assumed to be linear, with little investigation, if any, into the absolute or relative goodness of fit. The aim of the present work is to provide effective and accessible methods for a nonparametric estimation of the functional form of the predictor. These build on a) the strengths of the hidden Markov model (HMM) machinery (Zucchini and MacDonald 2009), in particular the forward algorithm, which allows for a simple and fast evaluation of the likelihood of a Markov-switching regression model (parametric or nonparametric), and b) the general advantages of penalized B-splines, i.e. P-splines (Eilers and Marx 1996), which we employ to obtain almost arbitrarily flexible functional estimators of the relationship between target variable and covariate(s). Model fitting is done via numerical maximum penalized likelihood estimation, using either generalized cross-validation or an information criterion approach to select smoothing parameters that control the balance between goodness-offit and smoothness. Since parametric polynomial models are included as limiting cases for very large smoothing parameters, this procedure also comprises the possibility to effectively reduce the functional effects to their parametric limiting cases, such that the conventional parametric Markov-switching regression models effectively are nested special cases of our more flexible models.

Our approach is by no means limited to models of the form given in (1). In fact, the flexibility of the HMM machinery allows for the consideration of models from a much bigger class, which we term Markov-switching generalized additive models (MS-GAMs). These are simply generalized additive models (GAMs) with an additional time component, where the predictor-including additive smooth functions of covariates, parametric terms and error terms-is subject to regime changes controlled by an underlying Markov chain, analogously to (1). While the methods do not necessitate a restriction to additive structures, we believe these to be most relevant in practice and hence have decided to focus on these models in the present work. Our work is closely related to that of Souza and Heckman (2014). Those authors, however, confine their consideration to the case of only one covariate and the identity link function. Furthermore, we note that our approach is similar in spirit to that proposed in Langrock et al. (2015), where the aim is to nonparametrically estimate the densities of the state-dependent distributions of an HMM.

The paper is structured as follows. In Sect. 2, we formulate general Markov-switching regression models, describe how to efficiently evaluate their likelihood, and develop the splinebased nonparametric estimation of the functional form of the predictor. The performance of the suggested approach is then investigated in three simulation experiments in Sect. 3. In Sect. 4, we demonstrate the feasibility and the potential of the approach by applying it (i) to advertising data and (ii) to Spanish energy price data. We conclude in Sect. 5.

\section{Markov-switching generalized additive models}

\subsection{Markov-switching regression models}

We begin by formulating a Markov-switching regression model with arbitrary form of the predictor, encompassing both parametric and nonparametric specifications. Let $\left\{Y_{t}\right\}_{t=1, \ldots, T}$ denote the target variable of interest (a time series), and let $x_{p 1}, \ldots, x_{p T}$ denote the associated values of the $p$ th covariate considered, where $p=1, \ldots, P$. We summarize the covariate values at time $t$ in the vector $\mathbf{x}_{\cdot t}=\left(x_{1 t}, \ldots, x_{P t}\right)$. Further let $s_{1}, \ldots, s_{T}$ denote the states of an underlying unobservable $N$-state Markov chain $\left\{S_{t}\right\}_{t=1, \ldots, T}$. Finally, we assume that conditional on $\left(s_{t}, \mathbf{x}_{\cdot}\right)$, $Y_{t}$ follows some distribution from the exponential family and is independent of all other states, covariates and observations. We write

$g\left(\mathbb{E}\left(Y_{t} \mid s_{t}, \mathbf{x}_{\cdot}\right)\right)=\eta^{\left(s_{t}\right)}\left(\mathbf{x}_{\cdot t}\right)$,

where $g$ is some link function, typically the canonical link function associated with the exponential family distribution considered. That is, the expectation of $Y_{t}$ is linked to the covariate vector $\mathbf{x}_{\cdot}$ via the predictor function $\eta^{(i)}$, which maps the covariate vector to $\mathbb{R}$, when the underlying Markov chain is in state $i$, i.e. $S_{t}=i$. Essentially there is one regression model for each state $i, i=1, \ldots, N$. In the following, we use the shorthand $\mu_{t}^{\left(s_{t}\right)}=\mathbb{E}\left(Y_{t} \mid s_{t}, \mathbf{x}_{\cdot t}\right)$.

To fully specify the conditional distribution of $Y_{t}$, additional parameters may be required, depending on the error distribution considered. For example, if $Y_{t}$ is conditionally Poisson distributed, then (2) fully specifies the statedependent distribution (e.g. with $g(\mu)=\log (\mu)$ ), whereas if $Y_{t}$ is normally distributed (in which case $g$ usually is the identity link), then the variance of the error needs to be specified, and would typically be assumed to also depend on the current state of the Markov chain. We use the notation $\phi^{\left(s_{t}\right)}$ to denote such additional state-dependent parameters (typically dispersion parameters), and denote the conditional density of $Y_{t}$, given $\left(s_{t}, \mathbf{x}_{t}\right)$, as $p_{Y}\left(y_{t}, \mu_{t}^{\left(s_{t}\right)}, \phi^{\left(s_{t}\right)}\right)$. The simplest and probably most popular such model assumes a conditional normal distribution for $Y_{t}$, a linear form of the predictor and a state-dependent error variance, leading to the model

$Y_{t}=\beta_{0}^{\left(s_{t}\right)}+\beta_{1}^{\left(s_{t}\right)} x_{1 t}+\cdots+\beta_{p}^{\left(s_{t}\right)} x_{P t}+\sigma_{s_{t}} \epsilon_{t}$, 
where $\epsilon_{t} \stackrel{i i d}{\sim} \mathcal{N}(0,1)$ (cf. Frühwirth-Schnatter 2006; Kim et al. 2008).

Assuming homogeneity of the Markov chain-which can easily be relaxed if desired-we summarize the probabilities of transitions between the different states in the $N \times N$ transition probability matrix (t.p.m.) $\boldsymbol{\Gamma}=\left(\gamma_{i j}\right)$, where $\gamma_{i j}=\operatorname{Pr}\left(S_{t+1}=j \mid S_{t}=i\right), i, j=1, \ldots, N$. The initial state probabilities are summarized in the row vector $\delta$, where $\delta_{i}=\operatorname{Pr}\left(S_{1}=i\right), i=1, \ldots, N$. It is usually convenient to assume $\delta$ to be the stationary distribution, which, if it exists, is the solution to $\boldsymbol{\delta} \boldsymbol{\Gamma}=\boldsymbol{\delta}$ subject to $\sum_{i=1}^{N} \delta_{i}=1$.

\subsection{Likelihood evaluation by forward recursion}

A Markov-switching regression model, with conditional density $p_{Y}\left(y_{t}, \mu_{t}^{\left(s_{t}\right)}, \phi^{\left(s_{t}\right)}\right)$ and underlying Markov chain characterized by $(\boldsymbol{\Gamma}, \boldsymbol{\delta})$, can be regarded as an HMM with additional dependence structure (here in the form of covariate influence); see Zucchini and MacDonald (2009). This opens up the way for exploiting the efficient and flexible HMM machinery. Most importantly, irrespective of the type of exponential family distribution considered, an efficient recursion can be applied in order to evaluate the likelihood of a Markov-switching regression model, namely the so-called forward algorithm. To see this, consider the vectors of forward variables, defined as the row vectors

$$
\begin{aligned}
& \boldsymbol{\alpha}_{t}=\left(\alpha_{t}(1), \ldots, \alpha_{t}(N)\right), t=1, \ldots, T \\
& \quad \text { where } \alpha_{t}(j)=p\left(y_{1}, \ldots, y_{t}, S_{t}=j \mid \mathbf{x} \cdot 1 \ldots \mathbf{x}_{\cdot t}\right) \\
& \quad \text { for } j=1, \ldots, N
\end{aligned}
$$

Here $p$ is used as a generic symbol for a (joint) density. Then the following recursive scheme can be applied:

$$
\begin{aligned}
\boldsymbol{\alpha}_{1} & =\delta \mathbf{Q}\left(y_{1}\right), \\
\boldsymbol{\alpha}_{t} & =\boldsymbol{\alpha}_{t-1} \mathbf{\Gamma Q}\left(y_{t}\right) \quad(t=2, \ldots, T),
\end{aligned}
$$

where

$\mathbf{Q}\left(y_{t}\right)=\operatorname{diag}\left(p_{Y}\left(y_{t}, \mu_{t}^{(1)}, \phi^{(1)}\right), \ldots, p_{Y}\left(y_{t}, \mu_{t}^{(N)}, \phi^{(N)}\right)\right)$.

The recursion (4) follows immediately from

$\alpha_{t}(j)=\sum_{i=1}^{N} \alpha_{t-1}(i) \gamma_{i j} p_{Y}\left(y_{t}, \mu_{t}^{(j)}, \phi^{(j)}\right)$,

which in turn can be derived in a straightforward manner using the model's dependence structure. Thus, the forward algorithm exploits the conditional independence assumptions to perform the likelihood calculation recursively, traversing along the time series and updating the likelihood and state probabilities at every step. The likelihood can then be written as a matrix product:

$\mathcal{L}(\boldsymbol{\theta})=\sum_{i=1}^{N} \alpha_{T}(i)=\delta \mathbf{Q}\left(y_{1}\right) \boldsymbol{\Gamma} \mathbf{Q}\left(y_{2}\right) \ldots \boldsymbol{\Gamma} \mathbf{Q}\left(y_{T}\right) \mathbf{1}$,

where $\mathbf{1} \in \mathbb{R}^{N}$ is a column vector of ones, and where $\boldsymbol{\theta}$ is a vector comprising all model parameters. The computational cost of evaluating (5) is linear in the number of observations, $T$, such that a numerical maximization of the likelihood is feasible in most cases, even for very large $T$ and moderate numbers of states $N$.

\subsection{Nonparametric modelling of the predictor}

Notably, the likelihood form given in (5) applies for any form of the conditional density $p_{Y}\left(y_{t}, \mu_{t}^{\left(s_{t}\right)}, \phi^{\left(s_{t}\right)}\right)$. In particular, it can be used to estimate simple Markov-switching regression models, e.g. with linear predictors, or in fact with any GLMtype structure within states. Here we are concerned with a nonparametric estimation of the functional relationship between $Y_{t}$ and $\mathbf{x}_{t}$. To achieve this, we consider a GAMtype framework (Wood 2006), with the predictor comprising additive smooth state-dependent functions of the covariates:

$$
\begin{aligned}
g\left(\mu_{t}^{\left(s_{t}\right)}\right)= & \eta^{\left(s_{t}\right)}\left(\mathbf{x}_{\cdot t}\right)=\beta_{0}^{\left(s_{t}\right)}+f_{1}^{\left(s_{t}\right)}\left(x_{1 t}\right)+f_{2}^{\left(s_{t}\right)}\left(x_{2 t}\right) \\
& +\cdots+f_{P}^{\left(s_{t}\right)}\left(x_{P t}\right) .
\end{aligned}
$$

We simply have one GAM associated with each state of the Markov chain. To achieve a flexible estimation of the functional form, we use penalised splines as introduced by Eilers and Marx (1996) (see also Fahrmeir et al. 2013, for an indepth discussion of penalised splines) and express each of the functions $f_{p}^{(i)}, i=1, \ldots, N, p=1, \ldots, P$, as a finite linear combination of a high number of B-spline basis functions, $B_{1}, \ldots, B_{K}$ :

$f_{p}^{(i)}(x)=\sum_{k=1}^{K} \gamma_{i p k} B_{k}(x)$.

Note that different sets of basis functions can be applied to represent the different functions, but to keep the notation simple we here consider a common set of basis functions for all $f_{p}^{(i)}$. B-splines have turned out to form a numerically stable, convenient basis for the space of polynomial splines, i.e. piecewise polynomials that are fused together smoothly at the interval boundaries; see Boor (1978) for more details. We use cubic B-splines, in ascending order in the basis used in (6), to obtain twice continuously differentiable function estimates. The number of B-splines involved in the specification of each of the functions, $K$, determines the flexibility of the functional form, as an increasing number 
of basis functions allows for an increasing curvature of the function being modeled. Instead of trying to select an optimal number of basis elements, we follow Eilers and Marx (1996) and modify the likelihood by including a difference penalty on coefficients of adjacent B-splines. The number of basis B-splines, $K$, then simply needs to be sufficiently large in order to yield high flexibility for the functional estimates. Once this threshold is reached, a further increase in the number of basis elements no longer changes the fit to the data due to the impact of the penalty. Considering second-order differences-which leads to an approximation of the integrated squared curvature of the function estimate (Eilers and Marx 1996) - leads to the difference penalty $0.5 \lambda_{i p} \sum_{k=3}^{K}\left(\Delta^{2} \gamma_{i p k}\right)^{2}$, where $\lambda_{i p} \geq 0$ are smoothing parameters and where $\Delta^{2} \gamma_{i p k}=\gamma_{i p k}-2 \gamma_{i p, k-1}+\gamma_{i p, k-2}$. Note that the integrated squared second derivative could of course also be evaluated explicitly for cubic B-splines. However, the approximation via a difference penalty allows to avoid the associated implementational costs at basically no cost in terms of the fit.

We then modify the (log-)likelihood of the MS-GAMspecified by $p_{Y}\left(y_{t}, \mu_{t}^{\left(s_{t}\right)}, \phi^{\left(s_{t}\right)}\right)$ in combination with (6) and underlying Markov chain characterized by $(\boldsymbol{\Gamma}, \boldsymbol{\delta})$-by including the above difference penalty, one for each of the smooth functions appearing in the state-dependent predictors:

$$
l_{\text {pen. }}(\boldsymbol{\theta})=\log (\mathcal{L}(\boldsymbol{\theta}))-\sum_{i=1}^{N} \sum_{p=1}^{P} \frac{\lambda_{i p}}{2} \sum_{k=3}^{K}\left(\Delta^{2} \gamma_{i p k}\right)^{2} .
$$

The maximum penalized likelihood estimate then reflects a compromise between goodness-of-fit and smoothness, where an increase in the smoothing parameters leads to an increased emphasis being put on smoothness. We discuss the choice of the smoothing parameters in more detail in Sect. 2.5. As $\lambda_{i p} \rightarrow \infty$, the corresponding penalty dominates the log-likelihood, leading to a sequence of estimated coefficients $\gamma_{i p 1}, \ldots, \gamma_{i p K}$ that are on a straight line. Thus, we obtain the common linear predictors, as given in (3), as a limiting case. Similarly, we can obtain parametric functions with arbitrary polynomial order $q$ as limiting cases by considering $(q+1)$ th order differences in the penalty. Thus, the common parametric regression models are essentially nested within the class of nonparametric models that we consider. One can of course obtain these nested special cases more directly, by simply specifying parametric rather than nonparametric forms for the predictor. On the other hand, it can clearly be advantageous not to constrain the functional form in any way a priori, though still allowing for the possibility of obtaining constrained parametric cases as a result of a datadriven choice of the smoothing parameters. Standard GAMs and even GLMs are also nested in the considered class of models $(N=1)$, but this observation is clearly less relevant, since powerful software is already available for these special cases.

\subsection{Inference}

For given smoothing parameters and given number of states, all model parameters - including the parameters determining the Markov chain, any dispersion parameters, the coefficients $\gamma_{i p k}$ used in the linear combinations of B-splines and any other parameters required to specify the predictor-can be estimated simultaneously by numerically maximizing the penalized log-likelihood given in (7). For each function $f_{p}^{(i)}$, $i=1, \ldots, N, p=1, \ldots, P$, one of the coefficients needs to be fixed to render the model identifiable, such that the intercept controls the height of the predictor function. A convenient strategy to achieve this is to first standardize each sequence of covariates $x_{p 1}, \ldots, x_{p T}, p=1, \ldots, P$, shifting all values by the sequence's mean and dividing the shifted values by the sequence's standard deviation, and second consider an odd number of B-spline basis functions $K$ with $\gamma_{i p,(K+1) / 2}=0$ fixed.

The numerical maximization is carried out subject to wellknown technical issues arising in all optimization problems, including parameter constraints and local maxima of the likelihood. The latter can be either easy to deal with or a challenging problem, depending on the complexity of the model considered. Numerical underflow (or overflow), which would typically arise for large $T$ if the likelihood itself was considered, is prevented via the consideration of the log-likelihood. Since the likelihood is a product of matrices, this requires the implementation of a scaling algorithm (for details, see, e.g., Zucchini and MacDonald 2009). Any suitable optimization routine can be applied to perform the likelihood maximization. In this work, we used $\mathrm{R}$ and the optimizer $\mathrm{nlm}$, which is a non-linear minimizer based on a Newton-type optimization routine. For more details on the algorithm, see Schnabel et al. (1985).

Uncertainty quantification, on both the estimates of parametric parts of the model and on the function estimates, can be performed based on the approximate covariance matrix available as the inverse of the observed Fisher information, or alternatively using a parametric bootstrap (Efron and Tibshirani 1993). The latter avoids relying on asymptotics, which is particularly problematic when the number of Bspline basis functions increases with the sample size. From the bootstrap samples, we can obtain pointwise as well as simultaneous confidence intervals for the estimated regression functions. Pointwise confidence intervals are simply given via appropriate quantiles obtained from the bootstrap replications. Simultaneous confidence bands are obtained by scaling the pointwise confidence intervals until they contain 
a pre-specified fraction of all bootstrapped curves completely (Krivobokova et al. 2010).

For the closely related class of nonparametric HMMs, identifiability holds under fairly weak conditions, which in practice will usually be satisfied, namely that the t.p.m. of the unobserved Markov chain has full rank and that the statespecific distributions are distinct (Gassiat et al. in press). This result transfers to the more general class of MS-GAMs if, additionally, the state-specific GAMs are identifiable. Conditions for the latter are simply the same as in any standard GAM. In particular, the nonparametric functions have to be centered around zero. Furthermore, in order to guarantee estimability of a flexible smooth function on a given domain, it is necessary that the covariate values cover that domain sufficiently well. In practice, i.e. when dealing with finite sample sizes, parameter estimation will be difficult if the level of correlation, as induced by the unobserved Markov chain, is low, and also if the state-specific GAMs are similar. The stronger the correlation in the state process, the clearer becomes the pattern and hence the easier it is for the model to allocate observations to states. Similarly, the estimation performance will be best, in terms of numerical stability, if the state-specific GAMs are clearly distinct. (See also the simulation experiments in Sect. 3 below.)

\subsection{Choice of the smoothing parameters}

In Sect. 2.4, we described how to fit an MS-GAM to data for a given smoothing parameter vector. To choose adequate smoothing parameters in a data-driven way, generalized cross-validation can be applied. A leave-one-out cross-validation will typically be computationally infeasible. Instead, for a given time series to be analyzed, we generate $C$ random partitions such that in each partition a high percentage of the observations, e.g. $90 \%$, form the calibration sample, while the remaining observations constitute the validation sample. For each of the $C$ partitions and any $\lambda=\left(\lambda_{11}, \ldots, \lambda_{1 P}, \ldots, \lambda_{N 1}, \ldots, \lambda_{N P}\right)$, the model is then calibrated by estimating the parameters using only the calibration sample (treating the data points from the validation sample as missing data, which is straightforward using the HMM forward algorithm; see Zucchini and MacDonald 2009). Subsequently, proper scoring rules (Gneiting and Raftery 2007) can be used on the validation sample to assess the model for the given $\lambda$ and the corresponding calibrated model. For computational convenience, we consider the loglikelihood of the validation sample, under the model fitted in the calibration stage, as the score of interest (now treating the data points from the calibration sample as missing data). From some pre-specified grid $\Lambda \subset \mathbb{R}_{>0}^{N \times P}$, we then select the $\lambda$ that yields the highest mean score over the $C$ cross-validation samples. The number of samples $C$ needs to be high enough to give meaningful scores (i.e. such that the scores give a clear pattern rather than noise only; from our experience, $C$ should not be smaller than 10 ), but must not be too high to allow for the approach to be computationally feasible.

An alternative, less computer-intensive approach for selecting the smoothing parameters is based on the Akaike Information Criterion (AIC), calculating, for each smoothing parameter vector from the grid considered, the following AIC-type statistic:

$\mathrm{AIC}_{p}=-2 \log \mathcal{L}+2 v$.

Here $\mathcal{L}$ is the unpenalized likelihood under the given model (fitted via penalized maximum likelihood), and $v$ denotes the effective degrees of freedom, defined as the trace of the product of the Fisher information matrix for the unpenalized likelihood and the inverse Fisher information matrix for the penalized likelihood (Gray 1992). Using the effective degrees of freedom accounts for the effective dimensionality reduction of the parameter space resulting from the penalization. From all smoothing parameter vectors considered, the one with the smallest $\mathrm{AIC}_{p}$ value is chosen.

\subsection{Choice of the number of states}

Choosing an appropriate number of states, $N$, is by no means a straightforward task. Even for the simpler parametric Markov-switching models, there is a variety of possible criteria for selecting $N$, including the AIC, the Bayesian Information Criterion, the Integrated Completed Likelihood criterion, the Hannan-Quinn criterion and cross-validated likelihood (see, e.g., Psaradakis and Spagnolo 2003; Celeux and Durand 2008), and it is our impression that most users pick their method of choice rather arbitrarily. For MS-GAMs, it is conceptually straightforward to choose $N$ for example based on cross-validated likelihood or on the AIC-type statistic (8). However, especially with information criteria, we have made the experience that these tend to favor overly complex state processes, often due to additional states being included simply to capture artefacts such as a few outlying observations. Thus, it is our view that one should not blindly trust these criteria when choosing $N$, and instead use them for guidance only. In addition, thoroughly checking the goodness of fit for different $N$ is important. In particular, this can help to (i) identify for which $N$ the fit is satisfactory, given the aim of the analysis (e.g. inference on the state-switching dynamics or prediction of future values), and (ii) reveal the sources of any lack of fit. Regarding (i), it is in our view often advisable to use a relatively small $N$ to guarantee computational tractability (e.g. if the state transition probabilities are functions of covariates) at the expense of a minor lack of fit. Regarding (ii), some typical problems of Markov-switching regression models are the following: 
- $N$ is too small;

- the distribution of the response variable is inadequate (e.g. due to overdispersion);

- the functional form of the predictor is not flexible enough.

While we would not generally claim that the choice of $N$ is easier for MS-GAMs than for the parametric counterparts, we do think that it is an advantage that the last of the three problems above can be excluded for MS-GAMs, since these models allow for arbitrary (smooth) functional forms (cf. Langrock et al. 2015). Thus, model checking for MS-GAMs centers around the autocorrelation and distribution of the residuals (the former to check the adequacy of the choice of $N$, the latter to check for a possible misspecification of the response distribution). Pseudo-residuals (also known as quantile residuals) allow for a comprehensive residual analysis in Markov-switching models (Zucchini and MacDonald 2009).

\section{Simulation experiments}

\subsection{Scenario I}

We first consider a relatively simple scenario, with a Poissondistributed target variable, a 2-state Markov chain selecting the regimes and only one covariate:

$Y_{t} \sim \operatorname{Poisson}\left(\mu_{t}^{\left(s_{t}\right)}\right)$

where

$\log \left(\mu_{t}^{\left(s_{t}\right)}\right)=\beta_{0}^{\left(s_{t}\right)}+f^{\left(s_{t}\right)}\left(x_{t}\right)$.

The functional forms of the predictors were chosen arbitrarily as

$f^{(1)}\left(x_{t}\right)=0.3 x_{t}^{2}+\sin \left(-x_{t}\right)$

and

$$
\begin{aligned}
f^{(2)}\left(x_{t}\right)= & -0.5-1.4 x_{t}+0.1 x_{t}^{2} \\
& +0.6 \sin \left(-x_{t}\right)+0.5 \cos \left(2 x_{t}\right)
\end{aligned}
$$

these functions are displayed by the dashed curves in Fig. 1. Both functions go through the origin. We further set $\beta_{0}^{(1)}=$ $\beta_{0}^{(2)}=2$ and

$$
\boldsymbol{\Gamma}=\left(\begin{array}{ll}
0.9 & 0.1 \\
0.1 & 0.9
\end{array}\right)
$$

All covariate values were drawn independently from a uniform distribution on $[-3,3]$. We ran 200 simulations, in each run generating $T=300$ observations from the model

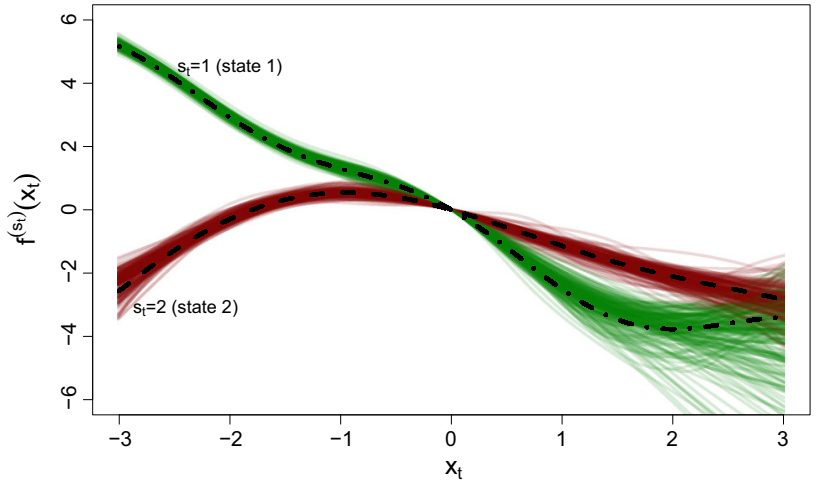

Fig. 1 Displayed are the true functions $f^{(1)}$ and $f^{(2)}$ used in Scenario I (dashed lines) and their estimates obtained in 200 simulation runs (green and red lines for states 1 and 2, respectively). (Color figure online)

described. An MS-GAM, with Poisson-distributed response and log-link, was then fitted via numerical maximum penalized likelihood estimation as described in Sect. 2.4 above. We set $K=15$, hence using 15 B-spline basis densities in the representation of each functional estimate.

We implemented both generalized cross-validation and the AIC-based approach for choosing the smoothing parameter vector from a grid $\Lambda=\Lambda_{1} \times \Lambda_{2}$, where $\Lambda_{1}=\Lambda_{2}=$ $\{0.125,1,8,64,512,4096\}$, considering $C=25$ folds in the cross-validation. For both approaches, we estimated the mean integrated squared error (MISE) for the two functional estimators, as follows:

$\widehat{\operatorname{MISE}}_{f(j)}=\frac{1}{200} \sum_{z=1}^{200}\left(\int_{-3}^{3}\left(\hat{f}_{z}^{(j)}(x)-f^{(j)}(x)\right)^{2} d x\right)$

for $j=1,2$, where $\hat{f}_{z}^{(j)}(x)$ is the functional estimate of $f^{(j)}(x)$ obtained in simulation run $z$. Using cross-validation, we obtained $\widehat{\operatorname{MISE}}_{f^{(1)}}=1.808$ and $\widehat{\operatorname{MISE}}_{f^{(2)}}=0.243$, while using the AIC-type criterion we obtained the slightly better values $\widehat{\operatorname{MISE}}_{f^{(1)}}=1.408$ and $\widehat{\operatorname{MISE}}_{f^{(2)}}=0.239$. In the following, we report the results obtained using the AICbased approach.

The sample mean estimates of the transition probabilities $\gamma_{11}$ and $\gamma_{22}$ were obtained as 0.894 (Monte Carlo standard deviation of estimates: 0.029$)$ and 0.896 (0.032), respectively. The estimated functions $\hat{f}^{(1)}$ and $\hat{f}^{(2)}$ from all 200 simulation runs are visualized in Fig. 1. The functions have been shifted so that they go through the origin. All fits are fairly reasonable. The sample mean estimates of the predictor value for $x_{t}=0$ were obtained as 2.002 (0.094) and 1.966 $(0.095)$ for states 1 and 2 , respectively.

\subsection{Scenario II}

The second simulation experiment we conducted is slightly more involved, with a normally distributed target variable, 


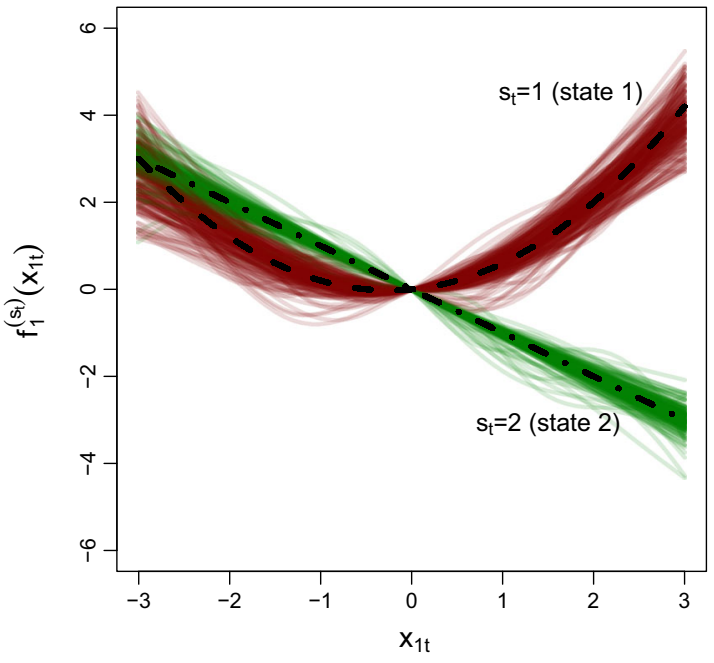

Fig. 2 Displayed are the true functions $f_{1}^{(1)}, f_{1}^{(2)}, f_{2}^{(1)}$ and $f_{2}^{(2)}$ used in Scenario II (dashed lines) and their estimates obtained in 200 simulation runs (red and green lines for states 1 and 2, respectively; $f_{1}^{(1)}$ and $f_{1}^{(2)}$, which describe the state-dependent effect of the covariate $x_{1 t}$ on the

an underlying 2-state Markov chain and now two covariates:

$Y_{t} \sim \mathcal{N}\left(\mu_{t}^{\left(s_{t}\right)}, \sigma_{s_{t}}\right)$,

where

$\mu_{t}^{\left(s_{t}\right)}=\beta_{0}^{\left(s_{t}\right)}+f_{1}^{\left(s_{t}\right)}\left(x_{1 t}\right)+f_{2}^{\left(s_{t}\right)}\left(x_{2 t}\right)$.

The functional forms were chosen as

$f_{1}^{(1)}\left(x_{1 t}\right)=0.2 x_{1 t}+0.4 x_{1 t}^{2}, \quad f_{1}^{(2)}\left(x_{1 t}\right)=-x_{1 t}$,

$f_{2}^{(1)}\left(x_{2 t}\right)=x_{2 t}+1.2 \sin \left(x_{2 t}\right) \quad$ and

$f_{2}^{(2)}\left(x_{2 t}\right)=-0.2 x_{2 t}-0.25 x_{2 t}^{2}-\sin \left(x_{2 t}\right)$;

see Fig. 2. Again all functions go through the origin. We further set $\beta_{0}^{(1)}=1, \beta_{0}^{(2)}=-1, \sigma_{1}=3, \sigma_{2}=2$ and

$\boldsymbol{\Gamma}=\left(\begin{array}{ll}0.95 & 0.05 \\ 0.05 & 0.95\end{array}\right)$

The covariate values were drawn independently from a uniform distribution on [ $-3,3]$. In each of 200 simulation runs, $T=1000$ observations were generated.

For the choice of the smoothing parameter vector, we considered the grid $\boldsymbol{\Lambda}=\Lambda_{1} \times \Lambda_{2} \times \Lambda_{3} \times \Lambda_{4}$, where $\Lambda_{1}=$ $\Lambda_{2}=\Lambda_{3}=\Lambda_{4}=\{0.25,4,64,1024,16384\}$. The AICbased smoothing parameter selection led to MISE estimates that overall were marginally lower than their counterparts obtained when using cross-validation $(0.555$ compared to 0.565 , averaged over all four functions being estimated), so again in the following we report the results obtained based on the AIC-type criterion. The (true) function $f_{1}^{(2)}$ is in fact

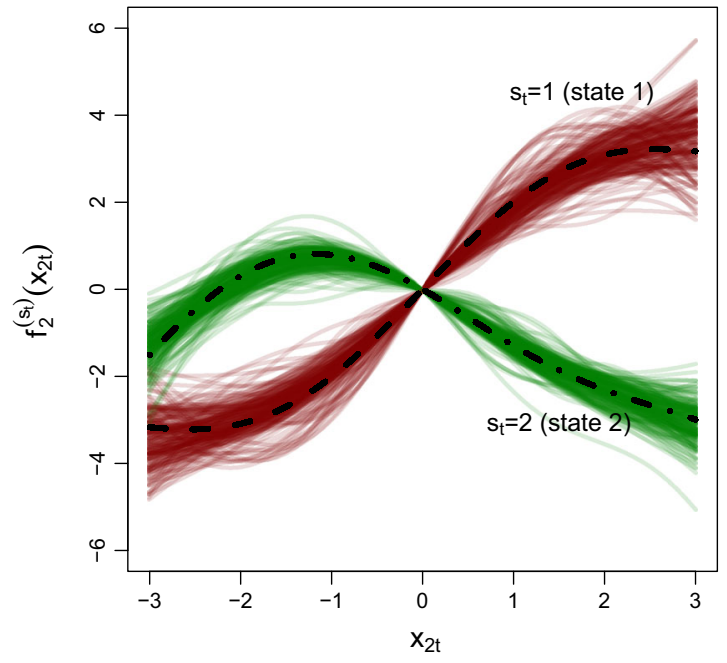

predictor, and corresponding estimates are displayed in the left panel; $f_{2}^{(1)}$ and $f_{2}^{(2)}$, which describe the state-dependent effect of the covariate $x_{2 t}$ on the predictor, and corresponding estimates are displayed in the right panel). (Color figure online)

a straight line, and, notably, the associated smoothing parameter was chosen as 16384 , hence as the maximum possible value from the grid considered, in 129 out of the 200 cases, whereas for example for the function $f_{2}^{(2)}$, which has a moderate curvature, the value 16384 was not chosen even once as the smoothing parameter.

In this experiment, the sample mean estimates of the transition probabilities $\gamma_{11}$ and $\gamma_{22}$ were obtained as 0.950 (Monte Carlo standard deviation of estimates: 0.011) and $0.948(0.012)$, respectively. The estimated functions $\hat{f}_{1}^{(1)}$, $\hat{f}_{1}^{(2)}, \hat{f}_{2}^{(1)}$ and $\hat{f}_{2}^{(2)}$ from all 200 simulation runs are displayed in Fig. 2. Again all have been shifted so that they go through the origin. The sample mean estimates of the predictor value for $x_{1 t}=x_{2 t}=0$ were $0.989(0.369)$ and $-0.940(0.261)$ for states 1 and 2, respectively. The sample mean estimates of the state-dependent error variances, $\sigma_{1}$ and $\sigma_{2}$, were obtained as $2.961(0.107)$ and $1.980(0.078)$, respectively. Again the results are very encouraging, with not a single simulation run leading to a complete failure in terms of capturing the overall pattern.

\subsection{Scenario III}

The estimator behavior both in Scenario I and in Scenario II is encouraging, and demonstrates that inference in MS-GAMs is clearly practicable in these two settings, both of which may occur in similar form in real data. However, as discussed in Sect. 2.4, in some circumstances, parameter identification in finite samples can be difficult, especially if the level of correlation as induced by the Markov chain is low. To illustrate this, we re-ran Scenario I, using the exact same configuration as described above except that we changed $\boldsymbol{\Gamma}$ to 


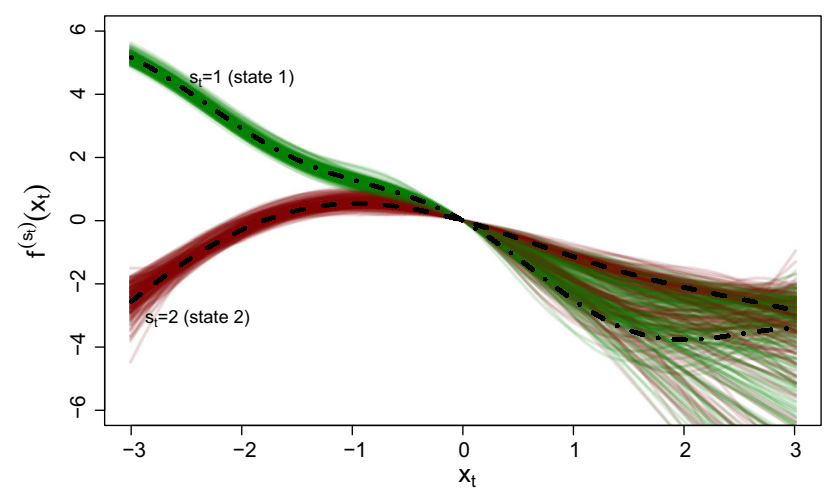

Fig. 3 Displayed are the true functions $f^{(1)}$ and $f^{(2)}$ used in Scenario III (dashed lines) and their estimates obtained in 200 simulation runs (green and red lines for states 1 and 2, respectively). (Color figure online)

$\boldsymbol{\Gamma}=\left(\begin{array}{ll}0.6 & 0.4 \\ 0.4 & 0.6\end{array}\right)$.

In other words, compared to Scenario I, there is substantially less autocorrelation in the series that are generated.

Figure 3 displays the estimated functions $\hat{f}^{(1)}$ and $\hat{f}^{(2)}$ in this slightly modified scenario. Due to the fairly low level of autocorrelation, the estimator performance is substantially worse than in Scenario I, and in several simulation runs the model failed to capture the overall pattern, by allocating pairs $\left(y_{t}, x_{t}\right)$ with high values of the covariate $x_{t}$ to the wrong state of the Markov chain. The deterioration in the estimator performance is also reflected by higher standard errors: The sample mean estimates of the transition probabilities $\gamma_{11}$ and $\gamma_{22}$ were obtained as 0.590 (Monte Carlo standard deviation of estimates: 0.082) and 0.593 (0.088), respectively, and the sample mean estimates of the predictor value for $x_{t}=0$ were obtained as $1.960(0.151)$ and $2.017(0.145)$ for states 1 and 2 , respectively.

\section{Real data examples}

\subsection{Advertising data}

We first consider a classic data set on Lydia Pinkham's annual sales and advertising expenditures during the period 19071960. The data set and its background are described in detail in Palda (1965). It comprises the sales in year $t, y_{t}$, and the annual advertising expenditures, $x_{t}$, of the company. Both figures are given in millions of U.S. dollars. The time series of annual sales displays two distinct peaks, in 1925 and 1945, respectively (see Fig. 1 in Palda 1965). Statistical analyses of such data can aid managers in determining the effectiveness of advertising (Smith et al. 2006).

As a baseline parametric Markov-switching model, we consider the model formulation suggested by Smith et al. (2006):

$$
\begin{aligned}
y_{t}= & \beta_{0}^{\left(S_{t}\right)}+\beta_{1}^{\left(S_{t}\right)} x_{t}+\beta_{2}^{\left(S_{t}\right)} y_{t-1} \\
& +\sigma_{s_{t}} \epsilon_{t}, \quad t=1908, \ldots, 1960,
\end{aligned}
$$

where $\epsilon_{t} \stackrel{i i d}{\sim} \mathcal{N}(0,1)$ and where $s_{t}$ is the state of a 2-state (stationary) Markov chain. This model, which involves 10 parameters, will be labeled MS-LIN in the following. Via numerical maximum likelihood, we obtained parameter estimates indicating that advertising was more effective in the model's state 1 than in state $2\left(\beta_{1}^{(1)}=0.746>0.397=\right.$ $\beta_{1}^{(2)}$ ), with state 2 involving a stronger carryover effect $\left(\beta_{2}^{(2)}=0.562>0.434=\beta_{2}^{(1)}\right)$. For the given model, the Viterbi algorithm allocated the years 1917-1924 and 19391944 to state 1 , and all other years to state 2 . The first switch to the state involving more effective advertising-i.e. the entry to state 1 in 1916/1917-followed the re-labeling of Lydia Pinkham's Vegetable Compound, which had been advertised as an almost universal remedy prior to 1914, and was now being sold primarily as a relief of "female troubles" (Palda 1965). A possible reason for the first departure from the state with the more effective advertising -i.e. the departure from state 1 in 1924/1925 — could be the fact that in 1925 Lydia Pinkham was ordered to stop advertising their Vegetable Compound as acting "directly upon female organs", such that they labeled it as "vegetable tonic" instead, which led to a drop in sales (Palda 1965). Similarly, the re-entering of state 2 in 1938/1939 could be related to the Federal Trade Commission re-allowing Lydia Pinkham to use their earlier, more effective marketing strategy in 1940. From 1946 onwards, sales plummeted due to a changed general perception of Lydia Pinkham as a "pseudoremedy from the previous century" (Applegate 2012), and this may explain the switch back to state 2 . These findings are notably different to those given in Smith et al. (2006), who reported only one state switch, such that their model's two regimes divided the data into a pre-war and a post-war period. We note that Smith et al. (2006) analyzed a slightly shorter data set, covering the period 1914-1960. However, we obtained different resultsvery similar to those reported here-also when fitting the model to that shorter series.

Next we fitted the following MS-GAM to the advertising data:

$$
\begin{gathered}
y_{t}=\beta_{0}^{\left(s_{t}\right)}+f^{\left(s_{t}\right)}\left(x_{t}\right)+\beta_{1}^{\left(S_{t}\right)} y_{t-1} \\
\quad+\sigma_{s_{t}} \epsilon_{t}, \quad t=1908, \ldots, 1960,
\end{gathered}
$$

again with $\epsilon_{t} \stackrel{i i d}{\sim} \mathcal{N}(0,1)$. This formulation is a semiparametric version of the general MS-GAM formulation, where we nonparametrically model the effect of the advertising expenditure $x_{t}$ but assume a simple linear effect of the previous year's sales, $y_{t-1}$, on the current year's sales, $y_{t}$. This model will be labeled MS-GAM in the following. We used this rel- 
Fig. 4 Lydia Pinkham data example: estimated state-dependent mean sales as functions of advertising expenditure (state 1 in green, state 2 in red), for the MS-LIN model (left plot) and for the MS-GAM (right plot).

Displayed are the predictor values when fixing the regressor $y_{t-1}$ at its overall mean, 1.84. (Color figure online)

Fig. 5 Lydia Pinkham data example: sample autocorrelation function and quantile-quantile plot (against the standard normal) of the forecast pseudo-residuals, for the fitted MS-LIN model (top plots) and for the fitted MS-GAM (bottom plots)
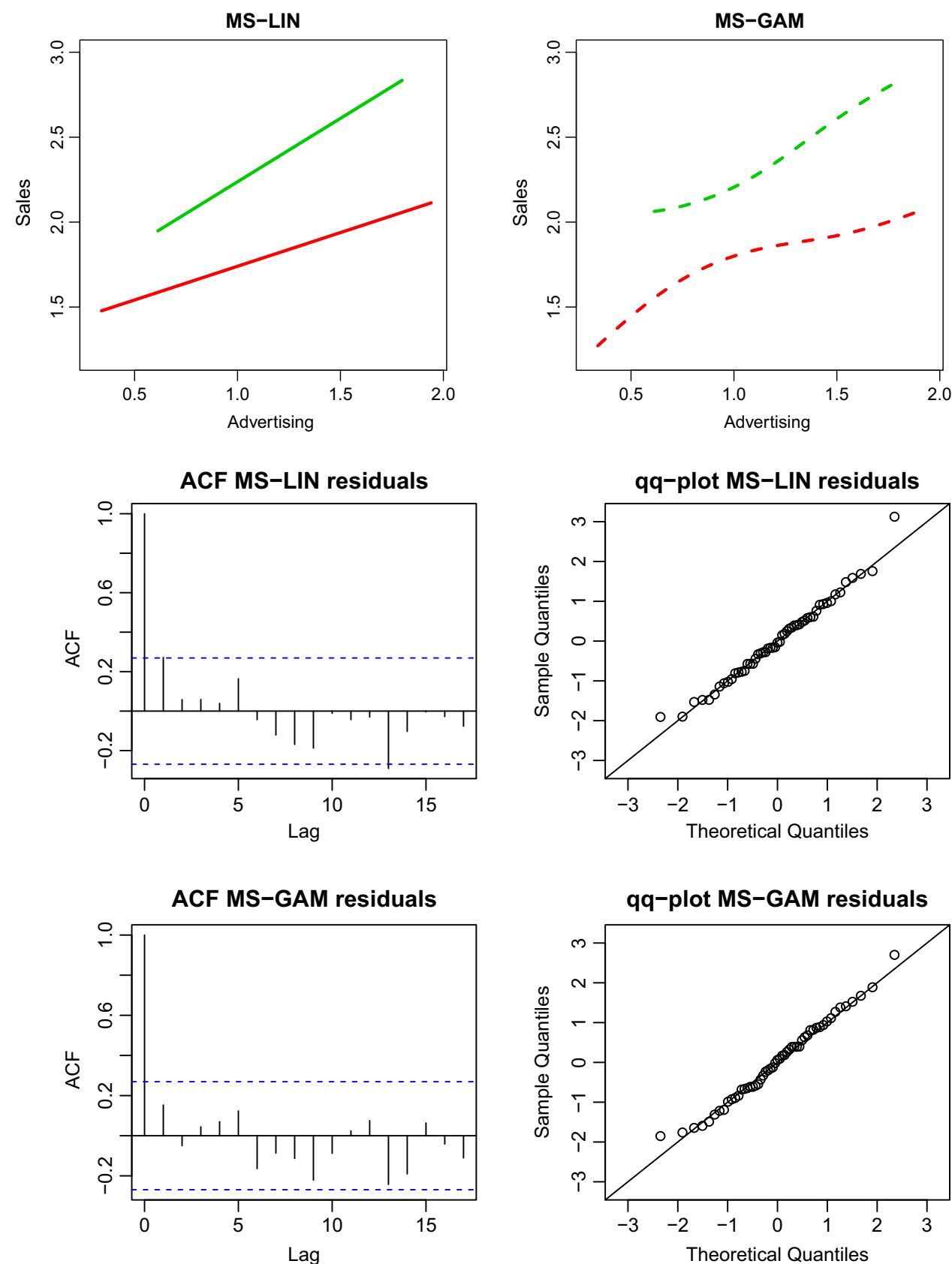

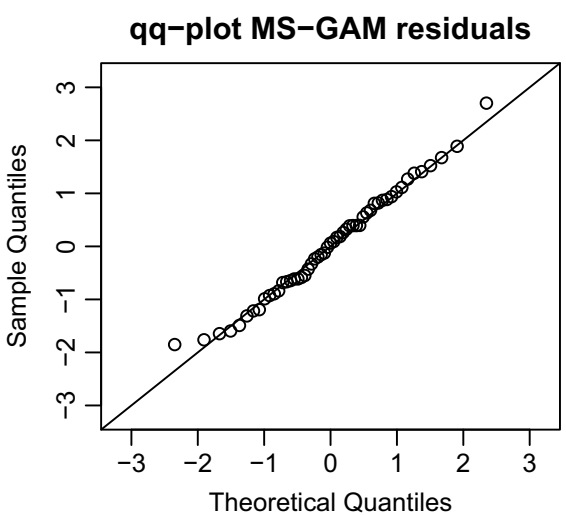

atively parsimonious model formulation as the fairly short time series does not contain sufficient information to fit the fully nonparametric model (i.e. also allowing for nonparametric effects of $y_{t-1}$ ).

Figure 4 compares the estimated effect of the advertising expenditure on the sales using the MS-LIN model and the MS-GAM, respectively. Overall, the regression structure within the two states is very similar for the two different models. In particular, for the MS-GAM considered, the Viterbi-decoded state sequence is identical to that obtained using the MS-LIN model. However, the more flexible MSGAM does reveal some interesting nuances. In particular, for state 2 there is a strong indication of a wearout effect in the benefits of advertising, suggesting that further increases of already high advertising expenditures do not increase sales as much as would be expected if a linear relation was assumed. This phenomenon is well-known in marketing (see, e.g., Corkindale and Newall 1978, Bass et al. 2007).

We additionally investigated the goodness of fit for the two models considered. Figure 5 displays the sample autocorrelation functions and quantile-quantile plots (against the standard normal) of the forecast pseudo-residuals obtained under the two different models (MS-LIN and MS-GAM). These pseudo-residuals, which can easily be obtained using 
Fig. 6 Spanish energy prices example: observed energy price against Euro/Dollar exchange rate (gray points), with estimated state-dependent mean energy prices (solid lines) for one-state (blue) and two-state (green and red) nonparametric and linear models; nonparametric models are shown together with associated approximate $95 \%$ pointwise confidence intervals obtained based on 999 parametric bootstrap samples (dotted lines). (Color figure online)
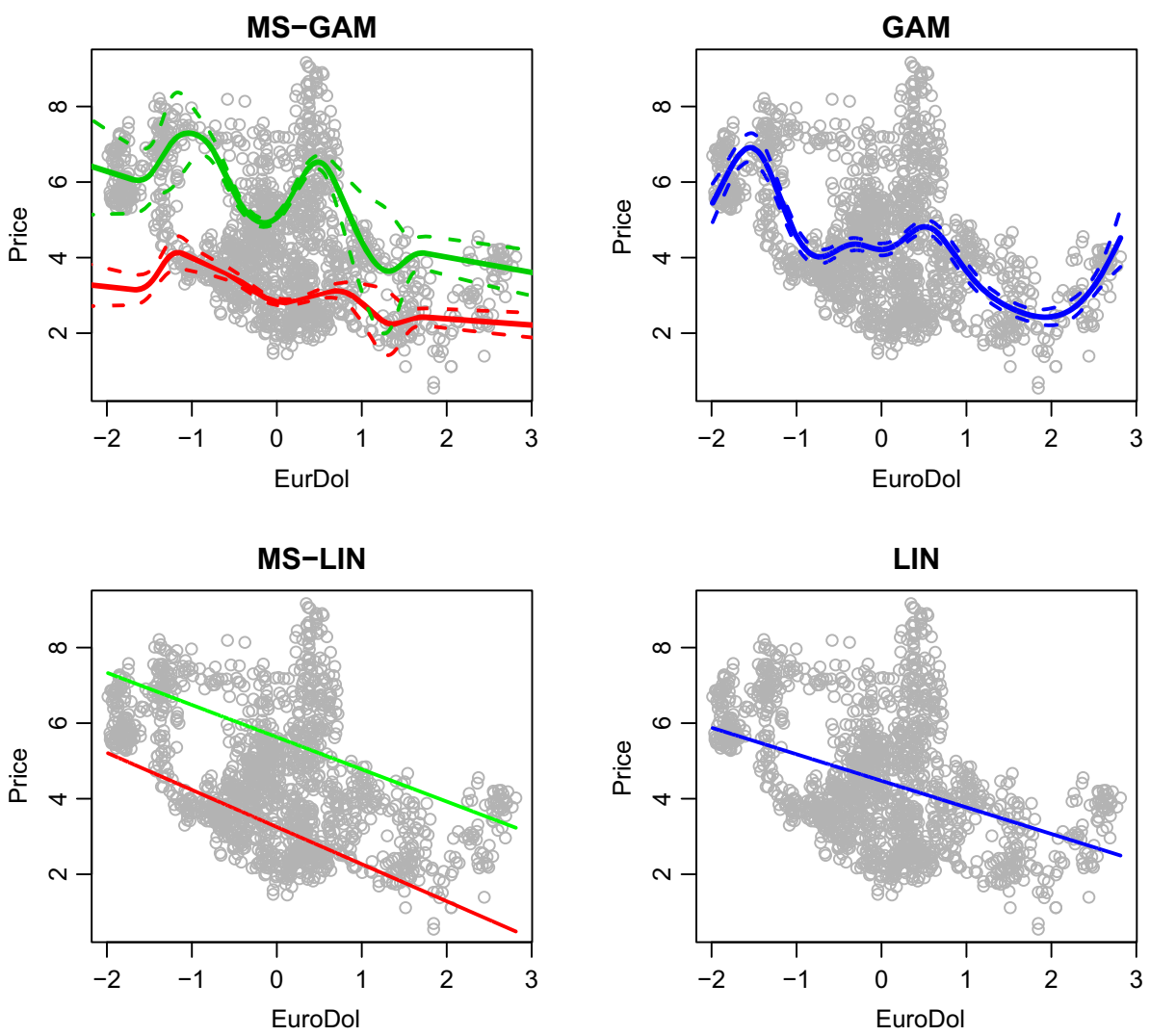

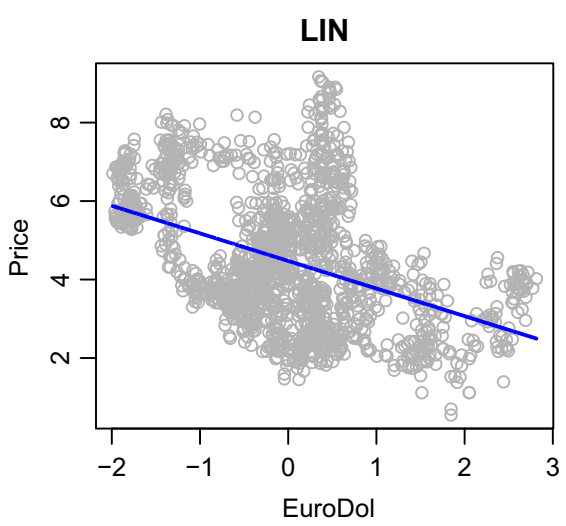

the forward variables, are approximately standard normally distributed if the model is adequate (Zucchini and MacDonald 2009). Overall, both models appear to fit the data adequately. However, for the parametric MS-LIN model there is some residual autocorrelation, which is often an indication that more states are required to fully capture the correlation structure of the time series (under the given model formulation). No such lack of fit is observed for the MSGAM.

\subsection{Spanish energy prices}

Next we analyze the data collected on the daily price of energy in Spain between 2002 and 2008. The data, 1784 observations in total, are available in the R package MSwM (Sanchez-Espigares et al. 2014). We consider the relationship over time between the price of energy, $y_{t}$, and the Euro/Dollar exchange rate, $x_{t}$. The commonly observed stochastic volatility of financial time series renders it unlikely that the relationship between these two variables is constant over time, and a possible, computationally efficient way to account for this is to consider a Markov-switching model. It is also probable that the two variables' unknown relationship within a regime has a non-linear functional form. As in the previous example, in the following we illustrate potential advantages of considering Markov-switching models with flexible nonparametric predictor functions, i.e. MS-GAMs, rather than GAMs or parametric Markov-switching models when analyzing time series regression data.

To this end, we consider four different models for the energy price data. As benchmark models, we considered two parametric models with state-dependent linear predictor $\beta_{0}^{\left(s_{t}\right)}+\beta_{1}^{\left(s_{t}\right)} x_{t}$, with one (LIN) and two states (MS-LIN), respectively, assuming the response variable $y_{t}$ to be normally distributed with state-dependent variance. Additionally, we considered two nonparametric models as introduced in Sect. 2.3, with one state (hence a basic GAM) and two states (MS-GAM), respectively. In these two models, we assumed $y_{t}$ to be gamma-distributed, applying the log link function to meet the range restriction for the (positive) mean.

Figure 6 shows the fitted curves for each model. For each one-state model (GAM and LIN), the mean curve passes through a region with no data (for values of $x_{t}$ around -1 ). This results in response residuals with clear systematic deviation. It is failings such as this which demonstrate the need for regime-switching models.

Models were also formally compared using an out-ofsample one-step-ahead forecast evaluation, by means of the sum of the log-likelihoods of observations $y_{u}$ under the models fitted to all preceding observations, $y_{1}, \ldots, y_{u-1}$, considering $u=501, \ldots, 1784$ (such that models are fitted to a reasonable number of observations). We obtained 


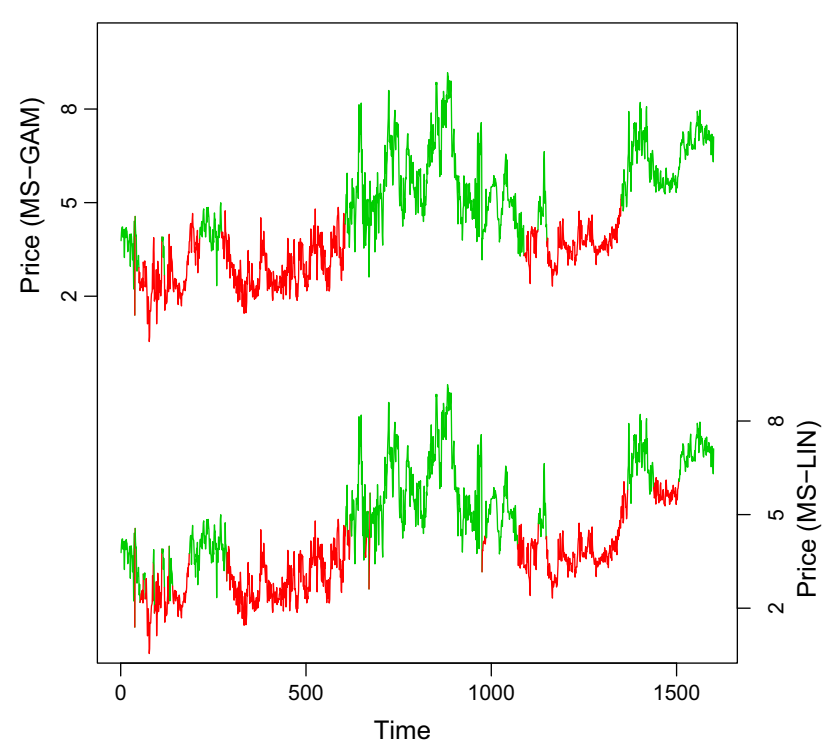

Fig. 7 Spanish energy prices example: globally decoded state sequence for the two-state (red and green) MS-LIN model and the two-state MS-GAM. (Color figure online)

the following log-likelihood scores for each model: -2314 for LIN, -2191 for GAM, -2069 for MS-LIN and -1703 for MS-GAM. Thus, in terms of out-of-sample forecasts, the MS-GAM performed much better than any other model considered. Both two-state models performed much better than the single-state models, however the inflexibility of the MSLIN model resulted in a poorer performance than that of its nonparametric counterpart, as clear non-linear features in the regression data are ignored.

For the MS-GAM, the transition probabilities were estimated to be $\gamma_{11}=0.991$ (standard error: 0.006) and $\gamma_{22}=$ 0.993 (0.003). The estimated high persistence within states gives evidence that identifiability problems such as those encountered in Scenario III in the simulation experiments did not occur here. Figure 7 gives the estimated regime sequence from the MS-GAM and the MS-LIN model obtained using the Viterbi algorithm. Both sequences are similar, with one state relating to occasions where price is more variable and generally higher. However, the MS-LIN model does tend to predict more changes of regime than the MS-GAM, which may be a result of its inflexibility.

While this second example is simplistic-for example, other explanatory covariates such as the oil price will also heavily affect the energy price-it nevertheless does illustrate the substantially increased flexibility, and hence increased potential to fit the data at hand, of MS-GAMs compared to their simpler parametric counterparts. At the very least, these models can prove useful as exploratory tools to identify key features in time series data with regime-switching patterns, without making any restrictive assumptions on the functional relationships a priori.

\section{Concluding remarks}

We have exploited the strengths of the HMM machinery and of penalized B-splines to develop a flexible new class of models, MS-GAMs, which show promise as a useful tool in time series regression analysis. A key strength of the inferential approach is ease of implementation, in particular the ease with which the code, once written for any MS-GAM, can be modified to allow for various model formulations. This makes interactive searches for an optimal model among a suite of candidate formulations practically feasible. Model selection, although not explored in detail in the current work, can be performed along the lines of Celeux and Durand (2008) using cross-validated likelihood, or can be based on AIC-type criteria such as the one we considered for smoothing parameter selection. For more complex model formulations, local maxima of the likelihood can become a challenging problem. In this regard, estimation via the EM algorithm, as suggested in Souza and Heckman (2014) for a smaller class of models, could potentially be more robust (cf. Bulla and Berzel 2008), but is technically more challenging, not as straightforward to generalize and hence less user-friendly (MacDonald 2014).

In the first example application, to advertising data, we demonstrated that the additional flexibility offered by MSGAMs can make an important difference regarding the exact quantification of the effect of some covariate (here: advertising expenditure) on some target variable (here: sales), in particular allowing to accurately quantify advertising wearout effects. In the second example application, to energy price data, the MS-GAM clearly outperformed the competing models in an out-of-sample comparison. This improvement is due to its accommodation of both the need for regime switches over time and the need to capture non-linear relationships within a regime. However, even the very flexible MS-GAM exhibited some shortcomings in this example. In particular, it is apparent from the plots, but also from the estimates of the transition probabilities, which indicated a very high persistence of regimes, that the regime-switching model addresses long-term dynamics, but fails to capture the short-term (day-to-day) variations within each regime. In this regard, it would be interesting to explore models that incorporate regime switching (for capturing long-term dynamics induced by persistent market states) but for example also autoregressive error terms within states (for capturing short-term fluctuations). Furthermore, the plots motivate a distributional regression approach, where not only the mean but also variance and potentially other parameters are modeled as functions of the covariates considered. In particular, it is conceptually straightforward to use the suggested type of estimation algorithm also for MS-GAMs for location, shape and scale (GAMLSS; Rigby and Stasinopoulos 2005). 
There are various other ways to modify or extend the approach, in a relatively straightforward manner, in order to enlarge the class of models that can be considered. First, as already seen in the application to advertising data, it is of course straightforward to consider semiparametric versions of the model, where some of the functional effects are modeled nonparametrically and others parametrically. Especially for complex models, with high numbers of states and/or high numbers of covariates considered, this can improve numerical stability and decrease the computational burden associated with the smoothing parameter selection. Second, the consideration of interaction terms in the predictor is possible via the use of tensor products of univariate basis functions. Third, the likelihood-based approach also allows for the consideration of more involved dependence structures (e.g. semi-Markov state processes; Langrock and Zucchini 2011). In particular, in the current model formulation we assume that a single univariate state process determines the GAM, such that changes in the state process affect all GAM parameters simultaneously. Conceptually there is no difficulty in devising models where different parts of the GAM are driven by different Markov state processes. However, with such models the dimensionality of the state process and hence the computational burden will increase rapidly. Finally, in case of multiple time series, random effects can be incorporated into a joint MS-GAM formulation.

Open Access This article is distributed under the terms of the Creative Commons Attribution 4.0 International License (http://creativecomm ons.org/licenses/by/4.0/), which permits unrestricted use, distribution, and reproduction in any medium, provided you give appropriate credit to the original author(s) and the source, provide a link to the Creative Commons license, and indicate if changes were made.

\section{References}

Applegate, E.: The Rise of Advertising in the United States: A History of Innovation to 1960. Scarecrow Press, Lanham (2012)

Bass, F.M., Bruce, N., Majumdar, S., Murthi, B.P.S.: Wearout effects of different advertising themes: a dynamic Bayesian model of the advertising-sales relationship. Mark. Sci. 26, 179-195 (2007)

Bulla, J., Berzel, A.: Computational issues in parameter estimation for stationary hidden Markov models. Comput. Stat. 13, 1-18 (2008)

Celeux, G., Durand, J.-P.: Selecting hidden Markov model state number with cross-validated likelihood. Comput. Stat. 23, 541-564 (2008)

Corkindale, D., Newall, J.: Advertising thresholds and wearout. Eur. J. Mark. 12, 329-378 (1978)

de Boor, C.: A Practical Guide to Splines. Springer, Berlin (1978)

de Souza, C.P.E., Heckman, N.E.: Switching nonparametric regression models. J. Nonparametric Stat. 26, 617-637 (2014)

Efron, B., Tibshirani, R.J.: An Introduction to the Bootstrap. Chapman $\&$ Hall/CRC, New York (1993)

Eilers, P.H.C., Marx, B.D.: Flexible smoothing with $B$-splines and penalties. Stat. Sci. 11, 89-121 (1996)

Fahrmeir, L., Kneib, T., Lang, S., Marx, B.: Regression: Models, Methods and Applications. Springer, Berlin (2013)
Frühwirth-Schnatter, S.: Finite Mixture and Markov Switching Models. Springer, New York (2006)

Gassiat, E., Cleynen, A., Robin, S.: Inference in finite state space non parametric Hidden Markov models and applications. Stat. Comput. (2015). doi:10.1007/s11222-014-9523-8

Gneiting, T., Raftery, A.E.: Strictly proper scoring rules, prediction, and estimation. J. Am. Stat. Assoc. 102, 359-378 (2007)

Goldfeld, S.M., Quandt, R.E.: A Markov model for switching regressions. J. Econom. 1, 3-16 (1973)

Gray, R.J.: Flexible methods for analyzing survival data using splines, with application to breast cancer prognosis. J. Am. Stat. Assoc. 87, 942-951 (1992)

Hamilton, J.D.: A new approach to the economic analysis of nonstationary time series and the business cycle. Econometrica 57, 357-384 (1989)

Hamilton, J.D.: Regime-switching models. In: Durlauf, S.N., Blume, L.E. (eds.) The New Palgrave Dictionary of Economics, 2nd edn. Palgrave Macmillan, New York (2008)

Kim, C.-J., Piger, J., Startz, R.: Estimation of Markov regime-switching regression models with endogenous switching. J. Econom. 143, 263-273 (2008)

Krivobokova, T., Kneib, T., Claeskens, G.: Simultaneous confidence bands for penalized spline estimators. J. Am. Stat. Assoc. 105, 852-863 (2010)

Langrock, R., Zucchini, W.: Hidden Markov models with arbitrary state dwell-time distributions. Comput. Stat. Data Anal. 55, 715-724 (2011)

Langrock, R., Kneib, T., Sohn, A., DeRuiter, S.L.: Nonparametric inference in hidden Markov models using P-splines. Biometrics 71, 520-528 (2015)

MacDonald, I.L.: Numerical maximisation of likelihood: a neglected alternative to EM? Int. Stat. Rev. 82, 296-308 (2014)

Palda, K.S.: The measurement of cumulative advertising effects. J. Bus. 38, 162-179 (1965)

Psaradakis, Z., Spagnolo, F.: On the determination of the number of regimes in Markov-switching autoregressive models. J. Time Ser. Anal. 24, 237-252 (2003)

Rigby, R.A., Stasinopoulos, D.M.: Generalized additive models for location, scale and shape. J. R. Stat. Soc. Ser. C 54, 507-554 (2005)

Sanchez-Espigares, J.A., Lopez-Moreno, A.: MSwM: Fitting MarkovSwitching Models. R package version 1.2. http://CRAN.R-project. org/package $=$ MSwM (2014)

Schnabel, R.B., Koontz, J.E., Weiss, B.E.: A modular system of algorithms for unconstrained minimization. ACM Trans. Math. Softw. 11, 419-440 (1985)

Smith, A., Naik, P.A., Tsai, C.-H.: Markov-switching model selection using Kullback-Leibler divergence. J. Econom. 134, 553-577 (2006)

Wang, P., Puterman, M.L.: Markov Poisson regression models for discrete time series. Part 1: Methodology. J. Appl. Stat. 26, 855-869 (2001)

Wood, S.: Generalized Additive Models: An Introduction with R. Chapman \& Hall/CRC, Boca Raton (2006)

Zucchini, W., MacDonald, I.L.: Hidden Markov Models for Time Series: An Introduction Using R. Chapman \& Hall/CRC, Boca Raton (2009) 